\title{
Visible Light Emission from Atomic Scale Patterns Fabricated by the Scanning Tunneling Microscope
}

Thirstrup, C.; Sakurai, M.; Stokbro, Kurt; Aono, M.

Published in:

Physical Review Letters

Link to article, DOI:

10.1103/PhysRevLett.82.1241

Publication date:

1999

Document Version

Publisher's PDF, also known as Version of record

Link back to DTU Orbit

Citation (APA):

Thirstrup, C., Sakurai, M., Stokbro, K., \& Aono, M. (1999). Visible Light Emission from Atomic Scale Patterns Fabricated by the Scanning Tunneling Microscope. Physical Review Letters, 82(6), 1241-1244.

https://doi.org/10.1103/PhysRevLett.82.1241

\section{General rights}

Copyright and moral rights for the publications made accessible in the public portal are retained by the authors and/or other copyright owners and it is a condition of accessing publications that users recognise and abide by the legal requirements associated with these rights.

- Users may download and print one copy of any publication from the public portal for the purpose of private study or research.

- You may not further distribute the material or use it for any profit-making activity or commercial gain

- You may freely distribute the URL identifying the publication in the public portal 


\title{
Visible Light Emission from Atomic Scale Patterns Fabricated by the Scanning Tunneling Microscope
}

\author{
C. Thirstrup, ${ }^{1,2}$ M. Sakurai,${ }^{1}$ K. Stokbro,${ }^{3}$ and M. Aono ${ }^{1,4}$ \\ ${ }^{1}$ The Institute of Physical and Chemical Research (RIKEN), Wako, Saitama 351-01, Japan \\ ${ }^{2}$ Core Research for Evolutional Science and Technology (CREST) Program, \\ Japan Science and Technology Corporation (JST), Saitama, Japan \\ ${ }^{3}$ Mikroelektronik Centret, DTU, Building 345, 2800 Lyngby, Denmark \\ ${ }^{4}$ Department of Precision Science and Technology, Osaka University, Suita, Osaka 565, Japan
}

(Received 3 August 1998)

\begin{abstract}
Scanning tunneling microscope (STM) induced light emission from artificial atomic scale structures comprising silicon dangling bonds on hydrogen-terminated $\mathrm{Si}(001)$ surfaces has been mapped spatially and analyzed spectroscopically in the visible spectral range. The light emission is based on a novel mechanism involving optical transitions between a tip state and localized states on the sample surface. The wavelength of the photons can be changed by the bias voltage of the STM. The spatial resolution of the photon maps is as good as that of STM topographic images and the photons are emitted from a quasipoint source with a spatial extension similar to the size of a dangling bond. [S0031-9007(98)08376-8]

PACS numbers: 73.20.At, 73.20.-r, 78.66. $-\mathrm{w}$
\end{abstract}

Nowadays, man-made structures can be created even on the atomic scale on semiconductor and metal surfaces by means of atom manipulation using the scanning tunneling microscope (STM) [1,2]. Such atomic scale structures designed appropriately should exhibit interesting electrical, optical, and magnetic properties as suggested by recent theoretical studies [3]. However, experimental observations of the properties of artificial atomic scale structures created by STM have been limited until now. Gimzewski et al. [4] and Berndt et al. [5] have pioneered another ability of the STM. Detecting photons emitted from the tunnel gap of the STM, they showed that light emission characteristics of sample surfaces can be obtained with atomic scale spatial resolution. In the present paper, we report for the first time light emission characteristics of artificial atomic scale structures fabricated by STM and observations of clear optical transitions of tunneling electrons between a tip state and localized energy levels on a sample surface. The model example of artificial atomic scale structures chosen in the present work is an array of silicon dangling bonds $(\mathrm{DBs})$ on hydrogen-terminated $\operatorname{Si}(001)-(3 \times 1)$ reconstructed surfaces $[\mathrm{Si}(001)-(3 \times 1)-\mathrm{H}$ surfaces]. The $\mathrm{Si}$ DBs are created with atomic resolution using the STM tip to desorb $\mathrm{H}$ atoms from the surfaces [2]. At both polarities of bias voltage, the Si DB sites exhibit a much stronger light emission in the visible spectral range than H-terminated Si sites, the spatial resolution of the light emission being comparable to that obtained in STM topographic images and the wavelength of the photons emitted can be changed by the bias voltage of the STM. The light emission does not involve diffusion of carriers or surface plasmon modes implying that the photons are emitted from a quasipoint source with a spatial extension comparable to the size of a DB.
The experiments were performed in a UHV chamber with a base pressure of $\sim 8 \times 10^{-9} \mathrm{~Pa}$ using an STM operated at room temperature. Electrolytically sharpened tungsten (W) tips and two types of $\mathrm{Si}(001)$ samples, an antimony-doped $\left(n=1 \times 10^{18} \mathrm{~cm}^{-3}\right)$ and a boron-doped $\left(p=1 \times 10^{18} \mathrm{~cm}^{-3}\right)$ sample, were used. The Si(001)$(3 \times 1)$-H surface was prepared by standard procedures [6]. The photons emitted from the tip of the STM were collected by an optical fiber bunch mounted in the UHV chamber and exhibiting constant optical transmission from 400 to $1100 \mathrm{~nm}$. The photons were counted using cooled photomultiplier tubes (PMTs). In order to obtain the spectral information, a Hamamatsu R636-10 PMT was used in combination with interference filters at five different wavelengths from 400 to $800 \mathrm{~nm}$ [photon energies $(h v)$ from 3.10 to $1.55 \mathrm{eV}$ ], each filter having a bandwidth of $40 \mathrm{~nm}$. The system response could be considered constant in the wavelength interval covered by the filters.

Figure 1(A) is a filled state STM topographic image of an atomic scale pattern of exposed DBs forming the letter "P" with a lateral size of $17 \mathrm{~nm}$ on the $n$-type $\mathrm{Si}(001)$ $(3 \times 1)$-H surface created by STM induced desorption of $\mathrm{H}$ atoms [2]. The STM image was recorded using normal constant current imaging conditions. Figure 1(B) shows the photon intensity as a function of the position of the STM (photon map [4,5]). The photon map was recorded at the same area as in Fig. 1(A) using the constant current scanning conditions: sample bias voltage $V_{b}=-3 \mathrm{~V}$, tunnel current $I_{t}=8 \mathrm{nA}$, and a slow scanning velocity $v_{s}=9 \mathrm{~nm} / \mathrm{s}$. Figure $1(\mathrm{C})$ is the STM topographic image recorded after the photon map with the same scanning conditions as in Fig. 1(A). From the photon map, it is observed that the exposed DBs forming the letter P exhibit a large photon intensity $\left(I_{\text {photon }}\right)[\sim 160$ counts $/ \mathrm{sec}(\mathrm{cps})$ 

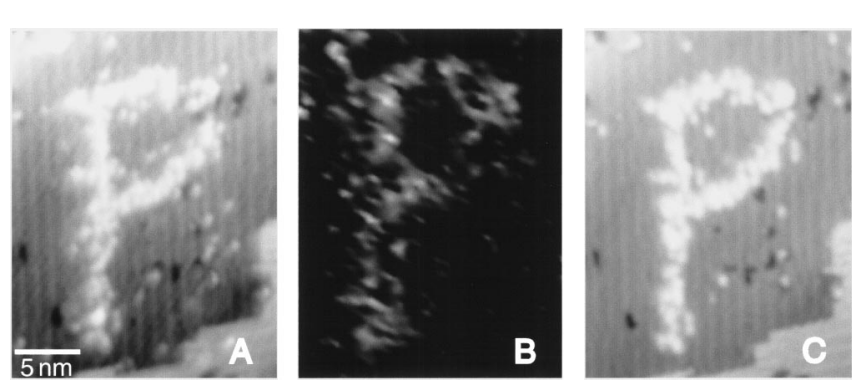

FIG. 1. (A) STM topographic filled state image of exposed dangling bonds forming the letter $\mathrm{P}$ on a $\mathrm{Si}(001)-(3 \times 1)-\mathrm{H}$ surface $\left(V_{b}=-2 \mathrm{~V}, I_{t}=0.2 \mathrm{nA}, v_{s}=1400 \mathrm{~nm} / \mathrm{s}\right.$; (B) the photon map recorded at the same area as in (A) using $V_{b}=$ $-3 \mathrm{~V}, I_{t}=8 \mathrm{nA}$, and $v_{s}=9 \mathrm{~nm} / \mathrm{s}$; and (C) STM topographic image recorded after the photon map and with the same scanning conditions as in (A). The later size of the letter $\mathrm{P}$ is $17 \mathrm{~nm}$.

in the bright regions in $\mathrm{B}$, while $I_{\text {photon }}$ from $\mathrm{H}$ terminated areas is much lower $(\sim 10 \mathrm{cps}$ in the dark regions in $\mathrm{B}$ ) and cannot be distinguished from the dark count level of similar magnitude. $I_{t}$ was stable during recording of the photon map excluding the possibility that the contrast in the photon map could be due to fluctuations in $I_{t}$. Comparing Figs. 1(A) and 1(C), it is noted that the letter P created by the STM is modified only slightly after recording the photon map at slow speed and fairly high $V_{b}$ and $I_{t}$. Note that the DB features in the photon map have as good a resolution as in the topographic images.

The photon map for the $n$-type sample shown in Fig. 1(B) was recorded at negative $V_{b}$; but for positive $V_{b}, \mathrm{H}$ desorption occurs at a much lower $I_{t}[2,7]$ where $I_{\text {photon }}$ would be too low to be detected with the present system. However, the desorption yield of deuterium (D) from D-terminated $\mathrm{Si}(001)$ surfaces is much lower than the corresponding desorption yield of $\mathrm{H}$ [8]. Using a positive $V_{b}=+3 \mathrm{~V}$ and $I_{t}=2 \mathrm{nA}$, we were able to obtain photon maps of exposed DBs on a $p$-type $\operatorname{Si}(001)-(3 \times 1)$-D surface with $I_{\text {photon }} \sim 130 \mathrm{cps}$ from DBs and $I_{\text {photon }} \sim 12 \mathrm{cps}$ from D-terminated areas.

In Fig. $2, I_{\text {photon }}$ from DB sites on $10 \times 10 \mathrm{~nm}^{2}$ squares of DBs created by the STM tip is plotted as a function of $V_{b}$ using $I_{t}=4 \mathrm{nA}$ and interference filters at five different values of $h v$ as indicated for (A)-(E) the $n$-type sample and $(\mathrm{F})-(\mathrm{J})$ the $p$-type sample. The circular symbols correspond to the experimental data and the solid curves are results of employing a least-squares smoothing filter to the data. The noise level is indicated by horizontal dashed lines and the vertical bars mark the threshold sample bias $\left(V_{b, \text { thres }}\right)$ where $I_{\text {photon becomes }}$ larger than the noise level. We have also measured $I_{\text {photon }}$ as a function of $I_{t}$ with $V_{b}$ being kept constant. For both types of samples and for both polarities of $V_{b}$, the results showed an approximately linear relationship between $I_{\text {photon }}$ and $I_{t}$, and the quantum efficiency $(\mathrm{QE})$ being almost independent of $I_{t}$ was estimated to be of the order of $10^{-6}$ photons/electron.

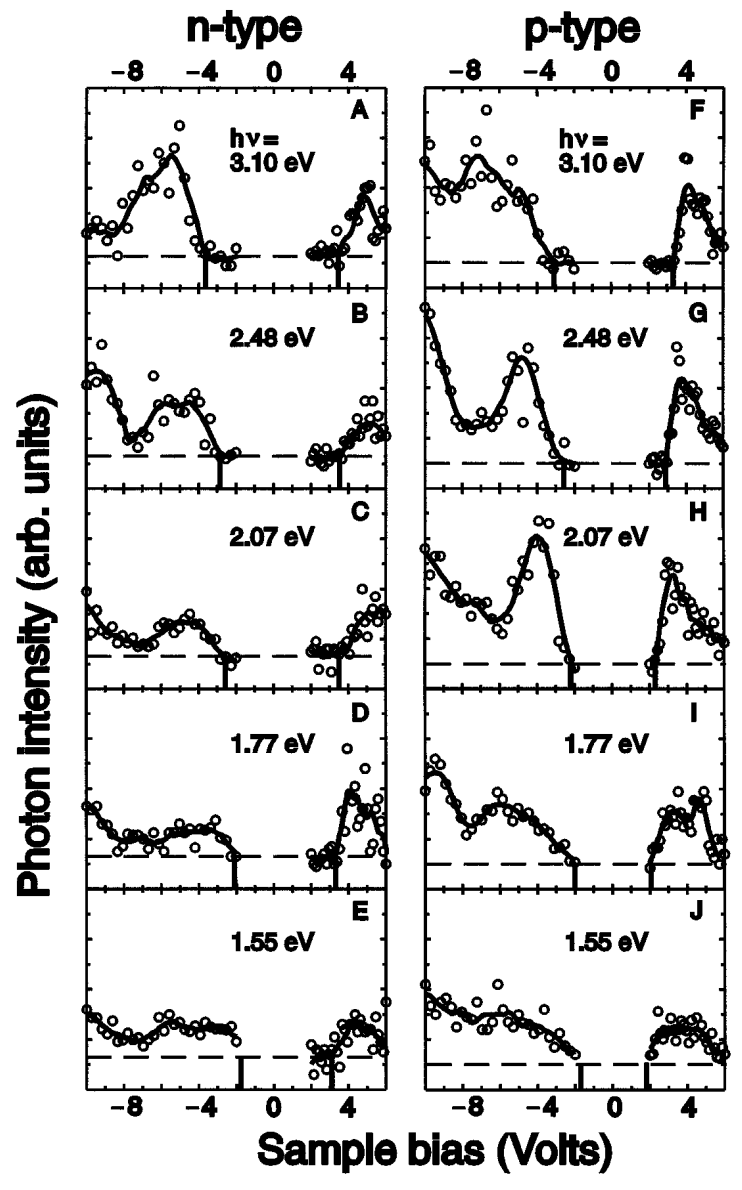

FIG. 2. STM induced photon intensity from dangling bond sites on $\mathrm{Si}(001)-(3 \times 1)-\mathrm{D}$ surfaces as a function of sample bias voltage $\left(I_{t}=4 \mathrm{nA}\right)$ at five different photon energies $(h v)$ as indicated for $(\mathrm{A})-(\mathrm{E})$ the $n$-type sample and $(\mathrm{F})-(\mathrm{J})$ the $p$-type sample. The solid curves are a five-point least-squares filter to the data. The horizontal dashed lines indicate the noise level, and the vertical bars mark the threshold sample bias where the photon intensity becomes larger than the noise level.

In Fig. $3, V_{b \text {,thres }}$ is plotted as a function of the energy of detected photons $(h v)$ for the $n$-type sample (open symbols) and the $p$-type sample (filled symbols). Thin solid lines going through the origin with slopes +1 and $-1 \mathrm{~V} / \mathrm{eV}$ are indicated in the upper part (positive $V_{b}$ ) and the lower part (negative $V_{b}$ ) of Fig. 3, respectively. This dependence of $V_{b \text {,thres }}$ upon $h v$ is expected if the light emission is caused by spatially indirect dipole transitions of tunneling electrons with an energy equal to the difference between the Fermi levels in the tip and in the sample. At both polarities of $V_{b}$ for the $p$-type sample, the experimental data approximately follow a linear relationship; but for the $n$-type sample, this is true only at negative $V_{b}$. At positive $V_{b}, V_{b}$,thres is $\sim 1.3 \mathrm{eV}$ larger for the $n$-type sample than for the $p$-type sample when $h v \leq 2 \mathrm{eV}$.

According to the experimental data presented in the present paper, the DB surface states must play an important role in the photon emission process at both polarities of $V_{b}$. The low $\mathrm{QE}$ of the photon emission process 


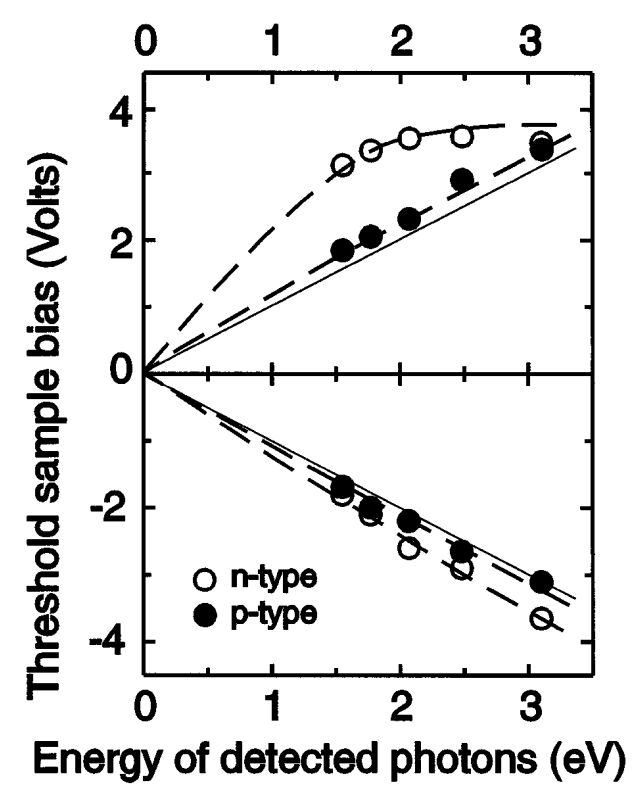

FIG. 3. Threshold sample bias obtained from the vertical bars in Fig. 2 as a function of the energy of detected photons for the $n$-type sample (open symbols) and the $p$-type sample (filled symbols). The two thin solid lines going through the origin have the slopes +1 and $-1 \mathrm{~V} / \mathrm{eV}$, respectively. The dashed curves are guides to the eye.

$\left(\sim 10^{-6}\right.$ photons/electron at both polarities of $\left.V_{b}\right)$ and the broad band emission from 1.55 to $3.1 \mathrm{eV}$ as observed in Fig. 2 make the possibility of band to band electron-hole pair recombination unlikely. Effects of surface plasmon modes can also be excluded, since the surface plasmon energy for $\mathrm{Si}(001)$ surfaces is $\sim 12 \mathrm{eV}$ [9], which is far away from the range covered by the experimental data set and a W tip exhibits no well-defined plasmon modes [10]. We also exclude optical transitions where an electron for $V_{b}>0$ or a hole for $V_{b}<0$ after tunneling through the tip-surface barrier causes an optical transition in the semiconductor via a dangling bond channel. At negative bias, only a small fraction of the hole current originates from low lying hole states [7], and in contrast to the experimental observations, QE would be much lower for $V_{b}<0$ than for $V_{b}>0$.

Other measurements show that QE is nearly independent of the tunnel current and the tip-sample distance. The electric field between the tip and the sample depends on the tip-sample distance implying that the polarization of a dangling bond also depends on this parameter. It is therefore unlikely that the dominant mechanism is scattering of tunneling electrons with the Coulomb field of charged DBs [11]. Instead we find that the experimental data can readily be interpreted in terms of spatially indirect dipole transitions between electronic states in the tip and DB states on the sample surface. From this mechanism, broad band photon spectra and similar QE at positive and negative $V_{b}$ are expected as observed experimentally. For a dipole transition between the tip and the sample, the emitted photons will mainly have a polarization perpendicular to the surface and with the wave vectors being parallel to the surface. The latter condition is important for the escape of the photon from the cavity between the tip and sample, since in a cavity between two metal surfaces with a separation less than $1 \mathrm{~nm}$ only photon modes with wave vectors parallel to the surfaces are allowed.

Schematic energy band diagrams can then be drawn as illustrated in Fig. 4 for a $p$-type semiconductor sample [Si(001)] and a metal tip (W) and $V_{b}>0(\mathrm{~A})$ and $V_{b}<0$ (B). In Figs. 4(A) and 4(B), the two Fermi levels $E_{F}$ and $E_{F}^{\prime}$ are indicated by dashed and solid lines, respectively. The bonding DB surface state $\pi$ is located at $0.2 \mathrm{eV}$ below the valence band maximum $E_{V}$, and the antibonding DB surface state $\pi^{*}$ is located at $0.4 \mathrm{eV}$ below the conduction band minimum $E_{C}$ [12]. In Figs. 4(C) and 4(D), $I_{\text {photon }}$ as obtained from Fig. 2 is plotted as a function of $h v$ for $V_{b}>0$ and $V_{b}<0$, respectively.

For positive $V_{b}$, the energy bands bend upwards as illustrated in Fig. 4(A). In the $I_{\text {photon }}$ spectra [see Fig. 4(C)], there is a peak at $h v \sim 2 \mathrm{eV}$ for $V_{b}=+3 \mathrm{~V}$. This peak is interpreted to be due to spatially indirect dipole transitions of tunneling electrons from a filled state close to $E_{F}^{\prime}$ in the tip to the empty $\pi^{*}$ state in the sample [see short arrow in Fig. 4(A)], since for $V_{b}=+3 \mathrm{~V}$, the $\pi^{*}$ state is located at $\sim 2 \mathrm{eV}$ below $E_{F}^{\prime}$ and $\sim 1 \mathrm{eV}$ above $E_{F}$. The position of the peak or equivalently the wavelength of the emitted photons can be changed by the bias voltage. When $V_{b}$ is increased to $+4 \mathrm{~V}$, the peak is shifted to $h v \sim 2.5-3 \mathrm{eV}$ (this shift is smaller than the increase in $e V_{b}$, since upward band bending increases with increasing $V_{b}$ ). The peak disappears for $V_{b}=+5 \mathrm{~V}$ and $+6 \mathrm{~V}$ where it is expected to be located outside the measuring range. Dipole

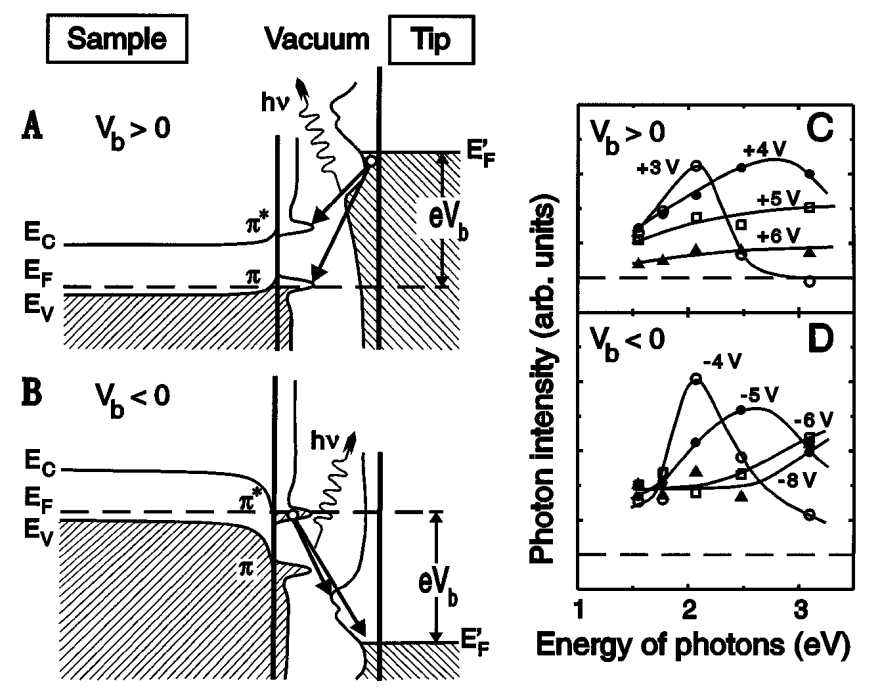

FIG. 4. Schematic energy band diagrams of the semiconductor sample, the vacuum and the metal tip regions in the case of a $p$-type $\mathrm{Si}(001)$ sample, (A) $V_{b}>0$ and (B) $V_{b}<0$. In (C) and (D), the corresponding photon intensities are plotted as a function of the energy of photons at various values of $V_{b}$ as indicated. The horizontal dashed lines in (C) and (D) indicate the noise level. 
transitions of tunneling electrons into an empty state positioned just above $E_{F}$ [see long arrow in Fig. 4(A)]

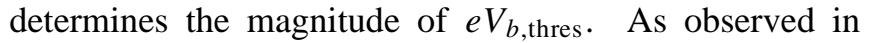
Figs. 2(F) $-2(\mathrm{~J})$, the $I_{\text {photon }}$ just above the positive $V_{b \text {,thres }}$ is large and exhibits a sharp peak for large values of $h v$ $(\geq 2.07 \mathrm{eV})$, but for small values of $h v(\leq 1.77 \mathrm{eV})$, the $I_{\text {photon }}$ is smaller and the spectra are more dull. This suggests that the $\pi$ state in Fig. 4(A) becomes empty and acts as the final state of optical transitions for large $V_{b}$ which causes a large band bending at the sample surface. For negative $V_{b}$, the energy bands bend downwards as illustrated in Fig. 4(B). In this case, the $I_{\text {photon }}$ spectra shown in Fig. 4(D) exhibit a peak at $\sim 2 \mathrm{eV}$ for $V_{b}=-4 \mathrm{~V}$. This peak is interpreted to be due to dipole transitions of tunneling electrons from a filled $\pi^{*}$ state close to $E_{F}$ in the sample into empty states in the tip (W) located at $\sim 2 \mathrm{eV}$ below $E_{F}$ (see short arrow); note that the density of empty states of $\mathrm{W}$ has a hump at $\sim 2 \mathrm{eV}$ above $E_{F}^{\prime}$ [13]. The peak is shifted to $h v \sim 2.5-3 \mathrm{eV}$ for $V_{b}=-5 \mathrm{~V}$ and disappears for $V_{b}=-6 \mathrm{~V}$ and $-8 \mathrm{~V}$ as expected. As observed in Figs. 2(F)-2(J), $I_{\text {photon }}$ just below the negative $V_{b \text {,thres }}$ is large exhibiting a sharp peak for large values of $h v(\geq 2.07 \mathrm{eV})$, but for small values of $h v(\leq 1.77 \mathrm{eV})$, $I_{\text {photon }}$ is smaller and the spectra are more dull. This is due to a fractional filling of the $\pi^{*}$ state at low $\left|V_{b}\right|$ where there is less band bending at the sample surface.

Energy band diagrams and $I_{\text {photon }}$ spectra similar to Fig. 4 can also be drawn for the $n$-type sample (not shown). However, since the $\pi$ state is below the valence band edge, it is unoccupied only at large fields [14]. For low $V_{b}$, electrons can decay only into the $\pi^{*}$ state, and this explains the $1.3 \mathrm{eV}$ difference in $V_{b \text {,thres }}$ between the $n$-type sample and the $p$-type sample at low biases as observed in Fig. 3. For $V_{b} \geq 3.2 \mathrm{~V}$, the field is strong enough to empty the $\pi$ state and this explains why $V_{b \text {,thres }}$ is the same for the $n$-type and the $p$-type samples for $h v \geq 3.2 \mathrm{eV}$. The variation in $h v$ as observed in Figs. 2(A)-2(E) is also consistent with this effect.

In conclusion, we have presented results of STM induced light emission from artificial atomic scale structures comprising exposed Si DBs on H-terminated $\mathrm{Si}(001)$ surfaces. At both polarities of bias voltage, DB sites yield a much stronger light emission in the visible spectral range than H-terminated sites. The light emission from DBs could be explained by dipole transitions between a tip state and $\mathrm{DB}$ states on the $\mathrm{Si}(001)$ surface, and the wave- length of the photons emitted could be changed by the bias voltage of the STM. Since the mechanism does not involve carrier diffusion or surface plasmon modes, the photons are emitted from a quasipoint source with an extension comparable to the size of a DB.

We thank M. Tsukada, A. P. Jauho, and K. Johnsen for helpful discussions. K. S. acknowledges support from the Danish Research Councils (STVF No. 9800466).

[1] J. A. Stroscio and D. M. Eigler, Science 254, 1319 (1991); I.-W. Lyo and Ph. Avouris, Science 253, 173 (1991); A. Kobayashi, F. Grey, R. S. Williams, and M. Aono, Science 259, 1724 (1993).

[2] T.-C. Shen, C. Wang, G. C. Abeln, J. R. Tucker, J. W. Lyding, Ph. Avouris, and R. E. Walkup, Science 268, 1590 (1995).

[3] S. Watanabe, Y. A. Ono, T. Hashizume, and Y. Wada, Phys. Rev. B 54, R17 308 (1996); R. Arita, K. Kuroki, H. Aoki, A. Yajima, and M. Tsukada, Phys. Rev. B 57, R6854 (1998); in Nanowires: Proceedings of NATO Advanced Research Workshop, Madrid, Spain, 1996, edited by P. A. Serena and N. Garcia (Kluwer Academic, Dordrecht, The Netherlands, 1997).

[4] J. K. Gimzewski, B. Reihl, J.H. Coombs, and R. R. Schlittler, Z. Phys. B 72, 497 (1988).

[5] R. Berndt, R. Gaisch, J. K. Gimzewski, B. Reihl, R. R. Schlittler, W. D. Schneider, and M. Tschudy, Science 262, 1425 (1993).

[6] J. J. Boland, Phys. Rev. Lett. 65, 3325 (1990).

[7] K. Stokbro, C. Thirstrup, M. Sakurai, U. Quade, Ben YuKuang Hu, F. Perez-Murano, and F. Grey, Phys. Rev. Lett. 80, 2618 (1998).

[8] Ph. Avouris, R. E. Walkup, A. R. Rossi, T.-C. Shen, G. C. Abeln, J. R. Tucker, and J. W. Lyding, Chem. Phys. Lett. 257, 148 (1996).

[9] A. J. Forsyth, A. E. Smith, and T. W. Josefsson, Surf. Sci. 357-358, 270 (1996).

[10] R. Berndt, J. K. Gimzevski, and P. Johansson, Phys. Rev. Lett. 71, 3493 (1993).

[11] W. Mönch, Phys. Status Solidi 36, 9 (1969).

[12] F. J. Himpsel and Th. Fauster, J. Vac. Sci. Technol. A 2, 815 (1984).

[13] I. R. Collins, A. D. Laine, and P. T. Andrews, J. Phys. C 4, 2891 (1992).

[14] M. McEllistrem, G. Haase, D. Chen, and R. J. Hamers, Phys. Rev. Lett. 70, 2471 (1993). 